\title{
How aging affects sleep-dependent memory consolidation?
}

\author{
Caroline Harand ${ }^{1,2,3,4}$, Françoise Bertran ${ }^{1,2,3,5}$, Franck Doidy 1,2,3,4, Fabian Guénolé ${ }^{1,2,3,6}$, \\ Béatrice Desgranges ${ }^{1,2,3,4}$, Francis Eustache ${ }^{1,2,3,4}$ and Géraldine Rauchs ${ }^{1,2,3,4}$ * \\ 1 INSERM, U1077, Caen, France \\ ${ }^{2}$ Université de Caen Basse-Normandie, UMR-S1077, Caen, France \\ ${ }^{3}$ Ecole Pratique des Hautes Etudes, UMR-S1077, Caen, France \\ ${ }^{4} \mathrm{CHU}$ de Caen, U 1077, Caen, France \\ ${ }^{5} \mathrm{CHU}$ de Caen, Unité d'évaluation et de traitement des troubles du sommeil, Caen, France \\ ${ }^{6} \mathrm{CHU}$ de Caen, Service de Psychiatrie de l'Enfant et de l'Adolescent, Caen, France
}

\section{Edited by:}

Philippe Peigneux, University Libre de Bruxelles, Belgium

\section{Reviewed by:}

Axel Hutt, INRIA CR Nancy, France

Timo Partonen, University of Helsinki,

Finland

\section{*Correspondence:}

Géraldine Rauchs, Unité de

Recherche U1077, INSERM, Ecole

Pratique des Hautes Etudes,

Université de Caen Basse-Normandie,

GIP Cyceron, Boulevard H. Becquerel BP 5229, 14074 Caen Cedex, France. e-mail: geraldine.rauchs@inserm.fr
Memories are not stored as they were initially encoded but rather undergo a gradual reorganization process, termed memory consolidation. Numerous data indicate that sleep plays a major role in this process, notably due to the specific neurochemical environment and the electrophysiological activity observed during the night. Two putative, probably not exclusive, models ("hippocampo-neocortical dialogue" and "synaptic homeostasis hypothesis") have been proposed to explain the beneficial effect of sleep on memory processes. However, all data gathered until now emerged from studies conducted in young subjects. The investigation of the relationships between sleep and memory in older adults has sparked off little interest until recently. Though, aging is characterized by memory impairment, changes in sleep architecture, as well as brain and neurochemical alterations. All these elements suggest that sleep-dependent memory consolidation may be impaired or occurs differently in older adults. This review outlines the mechanisms governing sleep-dependent memory consolidation, and the crucial points of this complex process that may dysfunction and result in impaired memory consolidation in aging.

Keywords: sleep, memory consolidation, aging, episodic memory, procedural memory, slow wave sleep, hippocampus
In humans, sleep is characterized by the cyclic occurrence of two main physiological stages, namely non-rapid eye movement (NREM) and rapid eye movement (REM) sleep. The former is usually subdivided in stage 1, stage 2, and slow wave sleep (SWS, stages 3 and 4) according to sleep depth. A sleep cycle lasts about 90-100 min, but SWS is most abundant during the first half of the night (up to $80 \%$ of sleep time), whereas REM sleep prevails in the second half of the night, alternating with stage 2 .

Likewise, memory is not a unitary phenomenon but is composed of multiple systems. In this review, we will focus on episodic and procedural memory, the two memory systems that were most investigated in this field. Episodic memory, a subcomponent of declarative memory, supports the encoding, storage, and retrieval of life events set in a specific spatiotemporal context. It relies upon the hippocampus and surrounding cortices, as well as the frontal and parietal cortices (Dickerson and Eichenbaum, 2010 for review). In contrast, procedural memory enables the gradual acquisition of skills through training and is not necessarily accessed consciously (non-declarative memory). This memory system mainly relies on striato-cortical networks (Doyon et al., 2003).

Ample evidence indicates that sleep is a physiological state favoring memory consolidation, the process by which recently acquired and labile memory traces are progressively strengthened into more permanent and/or enhanced forms. We will first see how sleep-dependent memory consolidation occurs in young healthy subjects and then examine the changes that may compromise this process in older adults.

\section{SLEEP-DEPENDENT MEMORY CONSOLIDATION IN YOUNG ADULTS}

Over the past two decades, numerous studies have provided evidence that sleep enhances memory consolidation. It has been initially shown that consolidation of declarative/episodic memories is enhanced by early SWS-rich periods of sleep whereas procedural memory rather benefits from late sleep during which REM sleep prevails (e.g., Plihal and Born, 1997). Other authors have pointed out the relevance of the succession of NREM-REM sleep cycles for memory consolidation regardless of the memory system the trace belongs to (Giuditta et al., 1995), potentially reflecting different processes operating on memory traces. Thus, Gais et al. (2000) showed that performance on a procedural visual discrimination task requires REM sleep but also a certain amount of preceding SWS. We also showed a complementary role of REM sleep and SWS in the consolidation of truly episodic memories. Whereas early SWS favors consolidation of the temporal aspects of episodic memories, REM sleep rather supports the consolidation of the spatial characteristics of these memories (i.e., location of words on a paper sheet) and the richness of contextual details (Rauchs et al., 2004). Consolidation of 
emotionally laden material also depends on REM sleep (Wagner et al., 2001).

More recently, studies have tried to unravel the electrophysiological substrates that mediate the beneficial effect of sleep on memory consolidation. Three main mechanisms may participate in this complex process of reorganization of memory traces during sleep: hippocampal reactivations, neocortical slow oscillations, and thalamo-cortical sleep spindles.

Using hippocampus-dependent spatial learning tasks in rodents, studies have reported that hippocampal place cells, activated during training, were reactivated in the same sequential order during subsequent periods of sleep (Wilson and McNaughton, 1994). Such neuronal reactivations were mainly observed during SWS (Skaggs and McNaughton, 1996; Kudrimoti et al., 1999) and more rarely during REM sleep (Louie and Wilson, 2001). These re-expressions are not limited to the hippocampus but also occur in the striatum and thalamus (Ribeiro et al., 2004) and in various neocortical areas (Qin et al., 1997; Ji and Wilson, 2007; Peyrache et al., 2009). This phenomenon was also observed in humans. Thus, Peigneux et al. (2004) showed that the hippocampus, activated during a spatial learning task, was reactivated during post-learning SWS. Interestingly, the amount of hippocampal activity during SWS was positively correlated with the overnight improvement of memory performance. More recently, Rasch et al. (2007) showed that reinstating during SWS an odor that was used as a contextual cue during learning, significantly enhanced episodic memory performance. This improvement was mediated by the hippocampus as odor re-exposure during SWS induced hippocampal activation that was even greater than during wakefulness.

Brain oscillations also participate actively in memory consolidation. Spindle density, spindle activity (index reflecting the activity or intensity of the spindle process), and the frequency of hippocampal ripples are increased during post-learning sleep (Fogel and Smith, 2011; Girardeau and Zugaro, 2011 for reviews). Learning experience increases the amplitude and slope of slow oscillations as well as slow wave activity during subsequent sleep (Diekelmann and Born, 2010 for review). These increases are associated to enhanced memory performance (e.g., Huber et al., 2004). In addition, inducing slow oscillation-like potential fields during post-learning sleep with transcranial direct current stimulation increases time spent in SWS, enhances EEG power in the slow oscillation frequency band and in the slow spindle band, and improves retention on an episodic memory task (Marshall et al., 2006).

\section{THE HIPPOCAMPAL-NEOCORTICAL DIALOGUE: A MODEL OF SLEEP-DEPENDENT MEMORY CONSOLIDATION}

The different findings cited above are summarized in a model of sleep-dependent consolidation of declarative/episodic memories termed the hippocampo-neocortical dialogue (Buzsáki, 1996; Figure 1A). Briefly, during wakefulness, freshly learned information is temporarily encoded into neocortical and hippocampal networks. During SWS, memory traces are repeatedly reactivated within hippocampal networks. Reactivations are associated with sharp wave-ripple activity in the hippocampus. They are driven by slow oscillations which synchronize reactivations with the occurrence of thalamo-cortical spindles to drive the transfer of memory traces toward neocortical sites where they will be stored durably. Low levels of cortisol and acetylcholine are required to allow the replay and transfer of information (Diekelmann and Born, 2010). The existence of this process has been demonstrated in rodents (Bontempi et al., 1999) and in humans using fMRI (Takashima et al., 2006). It occurs more so in subjects who slept after learning than in subjects who were sleep-deprived (Gais et al., 2007). With time, the ventromedial prefrontal cortex plays an important role in the retrieval of consolidated memories.

For procedural memories, the mechanism could be very similar, involving however different brain areas such as the striatum and cortico-cerebellar networks (Doyon and Benali, 2005).

Besides this active system consolidation hypothesis, an alternative mechanism has been proposed as the "synaptic homeostasis hypothesis" (Tononi and Cirelli, 2006). During wakefulness, encoding of new information leads to an increase in synaptic strength in the brain. Such a state is not sustainable in terms of energy and tissue volume demands, and saturates the capacity of synapses for

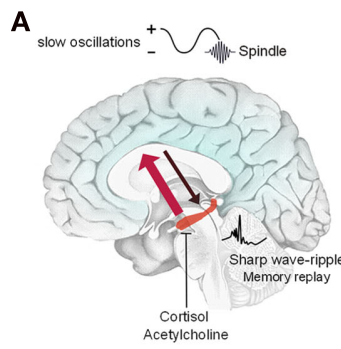

FIGURE 1 | Schematic representation of the possible alteration of sleep-dependent consolidation of declarative memories in older adults. (A) During wakefulness, information is encoded in neocortical and hippocampal networks (black arrow). During SWS, recently acquired information is repeatedly reactivated within hippocampal networks. Reactivations are associated with sharp waves-ripples and are driven by slow oscillations which also synchronize hippocampal memory reactivations with the occurrence of sleep spindles. These reactivations

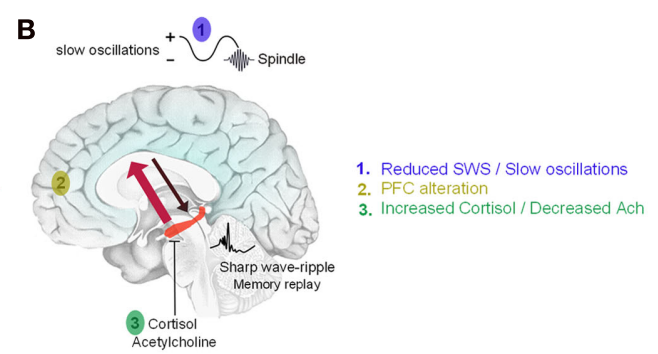

stimulate the transfer of memory traces toward neocortical sites for long term storage (red arrow). This transfer is allowed by low levels of acetylcholine and cortisol during early sleep. (B) With age, the decrease in SWS and slow oscillations combined with anatomical and functional changes in memory-related brain areas and neurochemical changes (acetylcholine, cortisol) are likely to explain that sleep-dependent memory consolidation is impaired in older adults. Adapted from Born et al. (2006). 
new learning. Sleep, and more precisely slow oscillations, would serve to downscale synaptic strength to a baseline level, keeping nevertheless a difference between synapses that were potentiated during prior waking and those that were not. This hypothesis has found experimental support in animals (Vyazovskiy et al., 2008; Bushey et al., 2011) and in humans (Huber et al., 2004; Van Der Werf et al., 2009).

\section{AGE-RELATED CHANGES IN SLEEP}

Sleep is substantially modified with increasing age. Older adults complain of early awakening in the morning and of difficulties to maintain continuous sleep. These difficulties are attested by an increase in the number of arousals during the night leading to sleep fragmentation. Total sleep time decreases, whereas time spent awake during the night or wake after sleep onset increase, thereby decreasing sleep efficiency (i.e., proportion of sleep time compared to time spent in bed). Sleep efficiency declines progressively with age, from approximately $86 \%$ at ages $37-54$ to $79 \%$ over age 70 (Bliwise, 2005). In contrast, the frequency of daytime naps increases with age (Humm, 2001), potentially worsening the disruption of nocturnal sleep.

The most striking change in sleep architecture is the dramatic decrease in time spent in SWS across the adult lifespan. This decline is further accompanied by a reduction in the number and amplitude of slow oscillations (Petit et al., 2004). To a lesser extent, changes of phasic events in stage 2, such as K-complexes and sleep spindles are also observed (Crowley et al., 2002). Changes concerning REM sleep tend to become significant only after age 50 . Thus, while the duration of REM sleep episodes increases through the night, it remains constant in older adults (Van Cauter et al., 2000), and the density of REMs is reduced (Darchia et al., 2003). Aging is further characterized by alterations of circadian rhythms (Bliwise, 2005) and reduced melatonin secretion (Pandi-Perumal et al., 2005). These two points may also have an impact on sleepdependent memory consolidation. Indeed, there is a circadian control of REM, but not of NREM sleep (Dijk and Czeisler, 1995; Dijk et al., 2000). Alterations of circadian rhythms may therefore result in changes in REM sleep. Melatonin is also intimately connected to memory consolidation as melatonin secretion is low during REM sleep (Birkeland, 1982) and high levels of this neurohormone inhibit memory formation during night (Rawashdeh et al., 2007).

\section{SLEEP-DEPENDENT CONSOLIDATION OF MEMORIES IN OLDER ADULTS}

Several elements suggest that sleep-dependent memory consolidation is altered with aging. First, older adults complain of memory impairment concerning mainly episodic memory. As mentioned above, consolidation of episodic memories relies upon SWS, which is also significantly reduced in older adults. Furthermore, with age some memory-related areas such as the frontal cortex undergo substantial structural and functional alterations (Kalpouzos et al., 2009). In contrast, the alteration of the hippocampus is more debated. This may be explained by the fact that the effects of age are not homogeneous within the hippocampus but rather concern its posterior part (Kalpouzos et al., 2009). More precisely, Mueller and Weiner (2009) reported a significant volume loss of CA3 (Mueller and Weiner, 2009). Albeit not consistently found (e.g., La Joie et al. (2010) who reported a strong effect of age on the volume of the subiculum only), this point is of particular interest since during SWS, freshly encoded memories are reactivated in the CA3 subfield and then spread back into CA1 and cortical areas for effective consolidation (Hasselmo, 1999). Evidence of impaired hippocampal reactivations after spatial learning has been provided in aged rats compared to young animals (Gerrard et al., 2008). In addition, frontal white-matter tracts are also affected by age and may lead to impaired interactions between the prefrontal cortex and the hippocampus (Salat et al., 2005).

Age-related changes in some neuroendocrine and neuromodulatory systems may likewise have an impact on sleep quality and architecture, and therefore on memory consolidation. Thus, aging is accompanied by a cholinergic hypofunction (Schliebs and Arendt, 2006). Classically, acetylcholine levels are high at the waking state - allowing encoding of new information - and reach a minimum during SWS. The nadir of acetylcholine during early sleep allows the reactivation of memory traces within hippocampal networks and their transfer toward neocortical areas (Hasselmo, 1999; Gais and Born, 2004). Age-related cholinergic hypofunction may not have consequences on SWS-dependent consolidation of declarative memories but could have repercussions on consolidation of procedural memories during REM sleep, during which acetylcholine levels are high. Indeed, cholinergic activation during REM sleep appears to be a critical factor mediating the beneficial effect of sleep on procedural memories (Rasch et al., 2009). Finally, aging is also accompanied by changes in the hypothalamo-pituitary-adrenal axis schematically leading to increases in evening cortisol levels (Buckley and Schatzberg, 2005). As memory consolidation requires low cortisol levels during early sleep (Plihal and Born, 1999) and since the hippocampus contains a high density of cortisol receptors (Roozendaal et al., 2009), elevated glucocorticoid levels may impair hippocampal functioning and impede the hippocampo-neocortical transfer.

All the points mentioned above seem closely interlinked and underline the complexity to assess sleep-dependent memory consolidation in older adults (Figure 1B). A handful of studies have been conducted in recent years and have provided mixed results (see also Pace-Schott and Spencer, 2011 for review).

The first studies conducted in healthy older adults (without depressive disorder or dementia) have mainly focused on REM sleep, due to the potential pharmacological modulations by cholinergic agents. Thus, Schredl et al. (2001) revealed positive correlations between overnight improvements on a word-pair recall task and REM sleep augmentation following administration of the cholinesterase inhibitor donepezil before bedtime during six consecutive nights. More recently, Hornung et al. (2007) assessed the effect of various REM sleep manipulations (increase or decrease in time spent in REM sleep) on episodic and procedural memory consolidation. REM sleep suppression was achieved by selective deprivation of this sleep stage. REM sleep augmentation was performed either physiologically through REM sleep rebound after a selective REM sleep deprivation or pharmacologically by administration of donepezil. All these manipulations had no effect on episodic memory. In contrast, only pharmacological REM 
sleep augmentation had positive effects on procedural memory consolidation, suggesting that cholinergic activation, more than REM sleep per se, is a crucial component of REM sleep memory consolidation in elderly people.

Other studies tried to relate memory performance with sleep stages in healthy older adults, without administration of cholinergic medication. Thus, Mazzoni et al. (1999) reported correlations between recall performance on a word-pair learning task and the mean duration of NREM/REM sleep cycles and the proportion of time spent in cycles, indicating that sleep structure and the cyclic succession of NREM-REM sleep are important for sleepdependent memory consolidation in older adults. More recently, Backhaus et al. (2007) reported a decline of sleep-dependent consolidation of semantically related word-pairs, associated with a decrease of early nocturnal SWS in 50 years old subjects. Retention of word-pairs after sleep was also positively correlated with the amount of SWS. These results were not replicated with lists of unrelated word-pairs (Wilson et al., in press). Finally, Aly and Moscovitch (2010) showed that memory of stories and personal events in older adults benefited from a night of sleep as much as in young adults. In addition, the number of hours slept was positively correlated with recall performance. The personal relevance of the material is therefore a crucial factor favoring sleep-dependent memory consolidation.

Concerning procedural memory, Spencer et al. (2007) have proposed to young and older adults a serial reaction time task. Performance was assessed after a 12-h interval filled with sleep or wakefulness. Older and young adults showed similar degrees of initial learning. However, performance of older adults did not improve following sleep, indicating that sleep-dependent consolidation is impaired with age. These results were replicated and extended to middle-aged subjects who showed, compared to young adults, a reduced sleep-dependent change in performance (Wilson et al., in press).

In another study, Peters et al. (2008) compared young and older healthy participants on a simple pursuit rotor task. Both age groups exhibited significant improvements after a 1-week delay but the magnitude of this improvement was lower in the older group. Interestingly, spindle density significantly increased after learning in young but not in old subjects. Finally, Tucker et al. (2011) were the only ones to observe, using a motor sequence task (fingertapping), similar improvements after sleep in young and older adults, although full expression of these improvements was seen

\section{REFERENCES}

Aly, M., and Moscovitch, M. (2010). The effects of sleep on episodic memory in older and younger adults. Memory 18, 327-334.

Backhaus, J., Born, J., Hoeckesfeld, R., Fokuhl, S., Hohagen, F., and Junghanns, K. (2007). Midlife decline in declarative memory consolidation is correlated with a decline in slow wave sleep. Learn. Mem. 14, 336-341.

Birkeland, A. J. (1982). Plasma melatonin levels and nocturnal

later during the retest session in older participants. As reported by Peters et al. (2008), no correlation between overnight improvement and sleep architecture or spindle density was observed in the elderly group.

Overall, studies conducted in older adults have provided mixed results, especially for declarative memory. While one may logically expect an impaired consolidation of declarative memories during early sleep, due to the dramatic decrease in SWS, this result was not consistently found. Qualitative differences between tasks may influence sleep's effect on memory processing. Further studies are needed to better understand the effects of age. As for procedural memory, studies report that sleep-dependent consolidation is impaired, or that the magnitude of overnight changes in performance is reduced or that the behavioral expression of consolidation is delayed compared to young adults. The reduction of cholinergic activation during REM sleep during aging is one of the mechanisms likely to account for these quantitative or qualitative changes in memory performance.

\section{CONCLUSION AND FUTURE DIRECTIONS}

Over the past 20 years, a plethora of studies have shown that sleep benefits memory consolidation in young healthy subjects. In older adults, several factors closely interlinked (decrease in SWS and slow oscillations, hypofunctioning and atrophy of frontal areas, reduction of the cholinergic tone, etc.) appear to hamper memory consolidation during sleep. Even if this field of research has sparked renewed interest during the last 5 years, some questions remain unanswered. In particular, studies combining sleep recordings, memory consolidation assessment, and functional neuroimaging acquisitions are needed to determine which brain areas will subserve the reorganization of memory traces during sleep and compensate for the frontal dysfunction. Studies in healthy older adults are also a prerequisite to understand how sleep-dependent memory consolidation is disrupted in neurological diseases such as Alzheimer's disease. Indeed, we observed in patients with mild Alzheimer's disease a specific decrease in the number and intensity of fast spindles (Rauchs et al., 2008) as well as a faster mean theta frequency during SWS (Hot et al., 2011). Both parameters were positively correlated with episodic memory performance confirming the deleterious impact of sleep changes on memory function and the existence of compensatory mechanisms in the early stages of the disease to maintain, albeit inefficiently, memory performance.

Born, J., Rasch, B., and Gais, S. (2006). Sleep to remember. Neuroscientist $12,410-424$.

Buckley, T. M., and Schatzberg, A. F. (2005). Aging and the role of the HPA axis and rhythm in sleep and memory-consolidation. Am. J. Geriatr. Psychiatry 13, 344-352.

Bushey, D., Tononi, G., and Cirelli, C. (2011). Sleep and synaptic homeostasis: structural evidence in Drosophila. Science 332, 1576-1581.

Buzsáki, G. (1996). The hippocamponeocortical dialogue. Cereb. Cortex 6, 81-92.
Crowley, K., Trinder, J., Kim, Y., Carrington, M., and Colrain, I. M. (2002). The effects of normal aging on sleep spindle and K-complex production. Clin. Neurophysiol. 113, 1615-1622.

Darchia, N., Campbell, I. G., and Feinberg, I. (2003). Rapid eye movement density is reduced in the normal elderly. Sleep 26, 973-977.

Dickerson, B. C., and Eichenbaum, H. (2010). The episodic memory system: neurocircuitry and disorders. Neuropsychopharmacology 35, 86-104. 
Diekelmann, S., and Born, J. (2010). The memory function of sleep. Nat. Rev. Neurosci.11, 114-126.

Dijk, D. J., and Czeisler, C. A. (1995). Contribution of the circadian pacemakerand the sleep homeostat to sleep propensity, sleep structure, electroencephalographic slow waves, and sleep spindle activity in humans. J. Neurosci. 15, 3526-3538.

Dijk, D. J., Duffy, J. F., and Czeisler, C. A. (2000). Contribution of circadian physiology nd sleep homeostasis to age-related changes in human sleep. Chronobiol. Int. 17, 285-311.

Doyon, J., and Benali, H. (2005). Reorganization and plasticity in the adult brain during learning of motor skills. Curr. Opin. Neurobiol. 15, 161-167.

Doyon, J., Penhune, V., and Ungerleider, L. G. (2003). Distinct contribution of the cortico-striatal and cortico-cerebellar systems to motor skill learning. Neuropsychologia 41, 252-262.

Fogel, S., and Smith, C. (2011). The function of the sleep spindle: a physiological index of intelligence and a mechanism for sleep-dependent memory consolidation. Neurosci. Biobehav. Rev. 35, 1154-1165.

Gais, S., Albouy, G., Boly, M., DangVu, T. T., Darsaud, A., Desseilles, M., Rauchs, G., Schabus, M., Sterpenich, V., Vandewalle, G., Maquet, P., and Peigneux, P. (2007). Sleep transforms the cerebral trace of declarative memories. Proc. Natl. Acad. Sci. U.S.A. 104, 18778-18783.

Gais, S., and Born, J. (2004). Low acetylcholine during slow-wave sleep is critical for declarative memory consolidation. Proc. Natl. Acad. Sci. U.S.A. 101, 2140-2144.

Gais, S., Plihal, W., Wagner, U., and Born, J. (2000). Early sleep triggers memory for early visual discrimination skills. Nat. Neurosci. 3 , 1335-1339.

Gerrard, J. L., Burke, S. N., McNaughton, B. L., and Barnes, C. A. (2008). Sequence reactivation in the hippocampus is impaired in aged rats. J. Neurosci. 28, 7883-7890.

Girardeau, G., and Zugaro, M. (2011). Hippocampal ripples and memory consolidation. Curr. Opin. Neurobiol. 21, 1-8.

Giuditta, A., Ambrosini, M. V., Montagnese, P., Mandile, P., Cotugno, M., Grassi Zucconi, G., and Vescia, S. (1995). The sequential hypothesis of the function of sleep. Behav. Brain. Res. 69, 157-166.
Hasselmo, M. E. (1999). Neuromodulation: acetylcholine and memory consolidation. Trends Cogn. Sci. (Regul. Ed.) 3, 351-359.

Hornung, O. P., Regen, F., DankerHopfe, H., Schredl, M., and Heuser, I. (2007). The relationship between REM sleep and memory consolidation in old age and effects of cholinergic medication. Biol. Psychiatry 61 750-757.

Hot, P., Rauchs, G., Bertran, F., Denise, P., Desgranges, B., Clochon, P., and Eustache, F. (2011). Changes in sleep theta rhythm are related to episodic memory impairment in early Alzheimer's disease. Biol. Psychol. 87, 334-339.

Huber, R., Felice, G. M., Massimini, M., and Tononi, G. (2004). Local sleep and learning. Nature 430, 78-81.

Humm, C. (2001). Sleep patterns in older people. Nurs. Times 97, 40-41.

Ji, D., and Wilson, M. A. (2007). Coordinated memory replay in the visual cortex and hippocampus during sleep. Nat. Neurosci. 10, 100-107.

Kalpouzos, G., Chételat, G., Baron, J. C., Landeau, B., Mevel, K., Godeau, C., Barré, L., Constans, J. M., Viader, F., Eustache, F., and Desgranges, B. (2009). Voxel-based mapping of brain gray matter volume and glucose metabolism profiles in normal aging. Neurobiol. Aging 30, 112-124.

Kudrimoti, H. S., Barnes, C. A., and McNaughton, B. L. (1999). Reactivation of hippocampal cell assemblies: effects of behavioral state, experience and EEG dynamics. J. Neurosci. 19, 4090-4101.

La Joie, R., Fouquet, M., Mézenge, F., Landeau, B., Villain, N., Mevel, K., Pèlerin, A., Eustache, F., Desgranges, B., and Chételat, G. (2010). Differential effect of age on hippocampal subfields assessed using a new high-resolution 3T MR sequence. Neuroimage 53, 506-514.

Louie, K., and Wilson, M. A. (2001). Temporally structured replay of awake hippocampal ensemble activity during rapid eye movement sleep. Neuron 29, 145-156.

Marshall, L., Helgadottir, H., Molle, M., and Born, J. (2006). Boosting slow oscillations during sleep potentiates memory. Nature 444, 610-613.

Mazzoni, G., Gori, S., Formicola, G., Gneri, C., Massetani, R., Murri, L., and Salzarulo, P. (1999). Word recall correlates with sleep cycles in elderly subjects. J. Sleep Res. 8, 185-188.

Mueller, S. G., and Weiner, M. W. (2009). Selective effect of age, Apo e4, and
Alzheimer's disease on hippocampal subfields. Hippocampus 19, 558-564.

Pace-Schott, E. F., and Spencer, R. M (2011). Age-related changes in the cognitive function of sleep. Prog. Brain Res. 191, 75-89.

Pandi-Perumal, S. R., Zisapel, N., Srinivasan, V., and Cardinali, D. P. (2005). Melatonin and sleep in aging population. Exp. Gerontol. 40 911-925.

Peigneux, P., Laureys, S., Fuchs, S., Collette, F., Perrin, F., Reggers, J., Phillips, C., Degueldre, C., Del Fiore, G., Aerts, J., Luxen, A., and Maquet, P. (2004). Are spatial memories strengthened in the human hippocampus during slow wave sleep? Neuron 44, 535-545.

Peters, K. R., Ray, L., Smith, V., and Smith, C. (2008). Changes in the density of stage 2 sleep spindles following motor learning in young and older adults. J. Sleep Res. 17, 23-33.

Petit, D., Gagnon, J. F., Fantini, M. L., Ferini-Strambi, L., and Montplaisir, J. (2004). Sleep and quantitative EEG in neurodegenerative disorders. $J$. Psychosom. Res. 56, 487-496.

Peyrache, A., Khamassi, M., Benchenane, K., Wiener, S. I., and Battaglia, F. P. (2009). Replay of rule-learning related neural patterns in the prefrontal cortex during sleep. Nat. Neurosci. 12, 919-926.

Plihal, W., and Born, J. (1997). Effects of early and late nocturnal sleep on declarative and procedural memory. J. Cogn. Neurosci. 9, 534-547.

Plihal, W., and Born, J. (1999). Memory consolidation in human sleep depends on inhibition of glucocorticoid release. Neuroreport 10 2741-2747.

Qin, Y. L., McNaughton, B. L., Skaggs, W. E., and Barnes, C. A. (1997). Memory reprocessing in corticocortical and hippocampo-cortical neuronal ensembles. Philos. Trans. R. Soc Lond. B Biol. Sci. 352, 1525-1533.

Rasch, B., Buchel, C., Gais, S., and Born, J. (2007). Odor cues during slow-wave sleep prompt declarative memory consolidation. Science 315 , 1426-1429.

Rasch, B., Pommer, J., Diekelmann, S., and Born, J. (2009). Pharmacological REM sleep suppression paradoxically improves rather impairs skill memory. Nat. Neurosci. 12, 396-397.

Rauchs, G., Bertran, F., Guillery-Girard, B., Desgranges, B., Kerrouche, N., Denise, P., Foret, J., and Eustache, F. (2004). Consolidation of strictly episodic memories mainly requires rapid eye movement sleep. Sleep 27, 395-401.
Rauchs, G., Schabus, M., Parapatics, S., Bertran, F., Clochon, P., Hot, P. Denise, P., Desgranges, B., Eustache, F., Gruber, G., and Anderer, P. (2008). Is there a link between sleep changes and memory in Alzheimer's disease? Neuroreport 19, 1159-1162.

Rawashdeh, O., de Borsetti, N. H., Roman, G., and Cahill, G. M. (2007). Melatonin suppresses nighttime memory formation in zebrafish. Science 318, 1144-1146.

Ribeiro, S., Gervasoni, D., Soares, E. S., Zhou, Y., Lin, S. C., Pantoia, J., Lavine, M., and Nicolelis, M. A. (2004). A long-lasting novelty induced neuronal reverberation during slow-wave sleep in multiple forebrain areas. PLoS Biol. 2, e24. doi:10.1371/journal.pbio.0020024

Roozendaal, B., McEwen, B. S., and Chattarji, S. (2009). Stress, memory and the amygdala. Nat. Rev. Neurosci. 10, 423-433.

Salat, D. H., Tuch, D. S., Hevelone, N. D., Fischl, B., Corkin, S., Rosas, H. D., and Dale, A. M. (2005). Agerelated changes in prefrontal white matter measured by diffusion tensor imaging. Ann. N. Y. Acad. Sci. 1064 , 37-49.

Schliebs, R., and Arendt, T. (2006). The significance of the cholinergic system in the brain during aging and in Alzheimer's disease. J. Neural Transm. 113, 1625-1644.

Schredl, M., Weber, B., Leins, M. L. and Heuser, I. (2001). Donepezilinduced REM sleep augmentation enhances memory performance in elderly, healthy persons. Exp. Gerontol. 36, 353-361.

Skaggs, W. E., and McNaughton, B. L. (1996). Replay of neuronal firing sequences in rat hippocampus during sleep following spatial experience. Science 271, 1870-1873.

Spencer, R. M., Gouw, A. M., and Ivry, R. B. (2007). Age-related decline of sleep-dependent consolidation. Learn. Mem. 14, 480-484.

Takashima, A., Petersson, K. M., Rutters, F., Tendolkar, I., Jensen, O., Zwarts, M. J., MacNaughton, B. L., and Fernandez, G. (2006). Declarative memory consolidation in humans: a prospective functional magnetic resonance imaging study. Proc. Natl. Acad. Sci. U.S.A. 103, 756-761.

Tononi, G., and Cirelli, C. (2006). Sleep function and synaptic homeostasis. Sleep Med. Rev. 10, 49-62.

Tucker, M., McKinley, S., and Stickgold, R. (2011). Sleep optimizes motor skill in older adults. J. Am. Geriatr. Soc. 59, 603-609.

Van Cauter, E., Leproult, R., and Plat, L. (2000). Age-related changes in slow 
wave sleep and REM sleep and relationship with growth hormone and cortisol levels in healthy men. JAMA 284, 861-868.

Van Der Werf, Y. D., Altena, E., Schoonheim, M. M., Sanz-Arigita, E. J., Vis, J. C., De Rijke, W., and Van Someren, E. J. (2009). Sleep benefits subsequent hippocampal functioning. Nat. Neurosci. 12, 122-123.

Vyazovskiy, V. V., Cirelli, C., PfisterGenskow, M., Faraguna, U., and Tononi, G. (2008). Molecular and electrophysiological evidence for net synaptic potentiation in wake and depression in sleep. Nat. Neurosci. 11, 200-208.

Wagner, U., Gais, S., and Born, J. (2001). Emotional memory formation is enhanced across sleep intervals with high amounts of rapid eye movement sleep. Learn. Mem. 8, 112-119. Wilson, J. K., Baran, B., Pace-Schott, E. F., Ivry, R. B., and Spencer, R. M. C. (in press). Sleep enhances declarative but not procedural learning in older adults. Neurobiol. Aging. [Epub ahead of print].

Wilson, M. A., and McNaughton, B. L. (1994). Reactivation of hippocampal ensemble memories during sleep. Science 265, 676-679.

Conflict of Interest Statement: The authors declare that the research was conducted in the absence of any commercial or financial relationships that could be construed as a potential conflict of interest.

Received: 07 November 2011; accepted: 10 January 2012; published online: 02 February 2012.

Citation: Harand C, Bertran F, Doidy F, Guénolé F, Desgranges B, Eustache F and
Rauchs $G$ (2012) How aging affects sleepdependent memory consolidation? Front. Neur. 3:8. doi: 10.3389/fneur.2012.00008 This article was submitted to Frontiers in Sleep and Chronobiology, a specialty of Frontiers in Neurology.

Copyright (c) 2012 Harand, Bertran, Doidy, Guénolé, Desgranges, Eustache and Rauchs. This is an open-access article distributed under the terms of the Creative Commons Attribution Non Commercial License, which permits noncommercial use, distribution, and reproduction in other forums, provided the original authors and source are credited. 